\title{
Prospective Volumetric Assessment of the Liver on a Personal Computer by Nonradiologists Prior to Partial Hepatectomy
}

\author{
Simon A. W. G. Dello $\cdot$ Jan H. M. B. Stoot $\cdot$ Rogier S. A. van Stiphout • \\ Johanne G. Bloemen · Stephen J. Wigmore · Cornelis H. C. Dejong • \\ Ronald M. van Dam
}

Published online: 7 December 2010

(c) The Author(s) 2010. This article is published with open access at Springerlink.com

\begin{abstract}
Background A small remnant liver volume is an important risk factor for posthepatectomy liver failure. ImageJ and OsiriX ${ }^{\circledR}$ are both free, open-source image processing software packages. The aim of the present study was to compare ImageJ and OsiriX ${ }^{\circledR}$ in performing prospective computed tomography (CT) volumetric analysis of the liver on a personal computer (PC) in patients undergoing major liver resection.

Methods Patients scheduled for a right hemihepatectomy were eligible for inclusion. Two surgeons and one surgical trainee measured volumes of total liver, tumor, and future resection specimen prospectively with ImageJ and OsiriX ${ }^{\circledR}$. A radiologist also measured these volumes with CT scanner-linked Aquarius iNtuition ${ }^{\circledR}$ software. Resection volumes were compared with the actual weights of the liver
\end{abstract}

S. A. W. G. Dello $(\bowtie) \cdot$ J. G. Bloemen ·

C. H. C. Dejong - R. M. van Dam

Department of Surgery, Maastricht University Medical Center,

P.O. Box 616, 6200 MD, Maastricht, The Netherlands

e-mail: S.dello@AH.unimaas.nl

J. H. M. B. Stoot

Department of Surgery, Orbis Medical Center,

Sittard, The Netherlands

R. S. A. van Stiphout

Department of Radiology, Maastricht University Medical

Center, Maastricht, The Netherlands

C. H. C. Dejong

Nutrim School for Nutrition, Toxicology, and Metabolism,

Maastricht University, Maastricht, The Netherlands

\section{S. J. Wigmore}

Department of Surgery, Royal Infirmary of Edinburgh,

Edinburgh, UK specimens removed during surgery, and differences between the measured liver volumes were analyzed.

Results A total of 15 patients ( 8 men, 7 women) with a median age of 63 years (48-79 years) were included. There was a significant correlation between the measured weights of resection specimens and the volumes calculated prospectively with ImageJ and $\operatorname{OsiriX}^{\circledR} \quad(r=0.89$; $r=0.83$, respectively). There was also a significant correlation between the volumes measured with radiological software iNtuition ${ }^{\circledR}$ and the volumes measured with ImageJ and OsiriX ${ }^{\circledR}(r=0.93 ; r=0.95$, respectively).

Conclusions There were no major differences in total liver volumes, resection volumes, or tumour volumes for these three software packages. Prospective hepatic CT volumetry with ImageJ or OsiriX ${ }^{\circledR}$ is reliable and can be accurately used on a PC by nonradiologists. ImageJ and OsiriX $^{\circledR}$ yield results comparable to the radiological software iNtuition ${ }^{\circledR}$.

\section{Introduction}

Hepatectomies are performed increasingly more often, mainly because indications for liver resection are continuously being extended. Whereas in the past a number of exclusion criteria were applied to the selection of patients for hepatectomy, they have now been largely abandoned, and postoperative remnant liver volume and function have become the main determinants of resectability [1-3].

Postoperative liver dysfunction may occur when the extent of tumor involvement requires major liver resection (three or more segments), leaving a small postoperative remnant liver [4-6]. A reliable volumetric assessment of the part of the liver to be resected and the future residual liver volume should be a critical part of preoperative 
evaluation, particularly for extended resections, to prevent postoperative liver failure after resection. The safety of liver resection may increase if an estimate of minimal remnant liver volume is obtained via computed tomography (CT) volumetry [4, 6-9]. This is especially important if the underlying liver parenchyma is abnormal or has impaired functional capacity. In this context, repeated liver resections, increasingly extensive resections, and resections after aggressive chemotherapy are likely to lead to more patients with small (functional) remnant livers and hence more risk of postoperative liver failure.

As we [4, 10-12] and others [7, 13, 14] have shown before, liver volumes can be calculated accurately from CT or magnetic resonance imaging (MRI) scans. The liberal use of existing professional image-processing software is often limited by costs, access, flexibility, lack of radiology staff, and specific hardware requirements. In addition, the intended operation and the potential surgical scenarios should be known to the investigator to predict the remnant liver volume accurately, which requires the expertise of a liver surgeon. The Digital Imaging and Communications in Medicine (DICOM) standard has enabled volumetry on a stand-alone or network-attached personal computer (PC) remote from the radiology hardware (CT or MRI scanner).

In 2007, our group conducted a retrospective study and proposed a method to perform liver volumetry with ImageJ [11], which is a free open-source image analysis software package developed at the National Institutes of Health (NIH) [8]. In 2010, our group assessed the accuracy of OsiriX $^{\circledR}$ for CT volumetry of the liver [12]. OsiriX ${ }^{\circledR}$ is a more user-friendly, freely downloadable open source image analysis software package for the Apple Mac OS. The accuracy of CT volumetry of the liver resection specimen prospectively prior to surgery has not yet been assessed for either of these software packages. Therefore, the objective of the present study was to compare ImageJ and OsiriX ${ }^{\circledR}$ when performing prospective CT volumetric analysis of the liver on a $\mathrm{PC}$ in patients undergoing major liver resection. The study also planned to compare the prospective measurements of ImageJ and OsiriX ${ }^{\circledR}$ to those of CT volumetry performed by a radiologist using classic radiology software (iNtuition ${ }^{\circledR}$ ) linked to a CT scanner system.

\section{Methods}

\section{Patients}

Patients with primary or secondary liver tumors in otherwise normal livers (mostly colorectal cancer liver metastases) planned for a right hemihepatectomy at Maastricht University Medical Center between January 2008 and April
2009 were eligible for inclusion in this study. All patients had a contrast enhanced CT scan in their routine preoperative assessment either in our hospital or in one of the surrounding university-affiliated district general teaching hospitals. Patients were admitted to the hospital 1 day preoperatively, and routine blood tests were performed.

\section{Surgical procedure}

Liver resection was performed as detailed elsewhere [15]. In each patient, laparotomy was performed by bilateral subcostal incision, followed by intraoperative ultrasonographic (US) assessment of the liver. Once resectability had been confirmed, appropriate mobilization of the liver was performed as a prelude to hepatic parenchymal transection, which was undertaken using a Cavitron Ultrasonic Surgical Aspirator (Force GSU System; Valleylab, Boulder, CO, USA). Argon beam coagulation (Erbe, Tübingen, Germany), clips, and sutures were used for hemostasis. Immediately after liver resection, the weights of the resected specimens were recorded in the operating theater with a Mettler Toledo scale.

\section{CT volumetry}

For volumetric analysis, four-phase CT scans were used that were provided on CD-ROM on one of two viewers: eFilm Lite (eFilm Medical, Toronto, Canada) or SIENET MagicView 300 VA42D (Siemens, Erlangen, Germany).

\section{Calculation of the liver volume using ImageJ}

ImageJ (version 1.33) was downloaded from http://rsb. info.nih.gov/ij/download.html (accession date 01-01-08). The portovenous phase of all contrast enhanced CT scans was used to facilitate optimal identification of liver segments and the anatomic resection plane. Details on how volumetry was performed with ImageJ have been described previously [11]. Briefly, the relevant source images were individually copied $(\mathrm{Ctrl}+\mathrm{V})$ from the $\mathrm{CT}$ viewer on the electronic patient care system and opened with ImageJ by dragging them to the ImageJ main window in the appropriate order. In cases of tertiary referred patients, relevant CT slices were searched in the original CT viewer on the CD-ROM. CT-slices on a CD-ROM were usually saved as compressed DICOM files, which cannot be opened or viewed via Windows Explorer. Therefore, the "Tudor DICOM Viewer Plug-in" was downloaded (free) from http://imagejdocu.tudor.lu/ and installed in the ImageJ menu. This plug-in allowed us to preview the relevant CT files and open them directly in ImageJ.

The total liver, tumor, and resected specimen were manually outlined. The respective region of interest (ROI) 
of each slice was added to the ROI manager. The area of each ROI was calculated and then multiplied by the slice thickness. Finally, all slice volumes were added up to calculate the volume of each three-dimensional structure (i.e., total liver, resected specimen, tumor).

Prospective volumetric analysis: ImageJ

Volumetric analysis using ImageJ was performed prospectively by two nonradiologists (S.A.W.G.D., J.H.M.B.S.) during the preoperative evaluation of the patient and was blinded to the operating surgeon. For definition of the anatomic segments, Couinaud's classification was used. In all right hemihepatectomies, transection followed Cantlie's line from the top of the gallbladder, paralleling the middle hepatic vein. straight to the suprahepatic inferior caval vein. In all patients, the middle hepatic vein remained in situ with the liver remnant. Consequently, during the venous phase, the middle hepatic vein on CT was used to perform the volumetric measurement.

To assess the accuracy of the volumetric measurements of the liver with ImageJ, the volumes of the resected specimens were compared with the actual weights of the specimens. (The weights of the specimens had been recorded in the operating theater immediately after resection.) The actual weights of the resection specimens remained blinded to the investigators conducting CT volumetry until all patients had been included and analyzed prospectively. The functional remnant liver volume (FRLV) percentage was calculated with the following formula. FRLV [\%] = $100 \% *$ [total liver volume - resected volume] / [total liver volume - tumour liver volume]

\section{Prospective volumetric analysis: OsiriX ${ }^{\circledR}$}

The 32-bit OsiriX ${ }^{\circledR}$ version 3.3 was downloaded from: http://www.osirix-viewer.com. A 2.8-GHz Intel Core 2 Duo 24" iMac (Apple, Cupertino, CA, USA) was used. Details on how volumetry was performed with Osiri ${ }^{\circledR}$ have been described previously [12]. Volumes calculated with ImageJ were compared to the volumes obtained by CT volumetry using OsiriX ${ }^{\circledR}$ by one of the liver surgeons (R.M.vanD.).

\section{Comparison of ImageJ and OsiriX ${ }^{\circledR}$ with iNtuition ${ }^{\circledR}$}

Volumes calculated with ImageJ and OsiriX ${ }^{\circledR}$ were compared to the volumes obtained by CT volumetry performed by a specialized liver radiologist (R.S.A.vanS.) using radiology image analysis software from TeraRecon linked to an Aquarius iNtuition ${ }^{\circledR}$ workstation. Volumetric analysis performed with ImageJ and OsiriX ${ }^{\circledR}$ was blinded to the radiologist. The volume of the liver resection specimen measured by the radiologist was used as a second standard of reference instead of the actual weight of the resected specimen.

\section{Reliability: ImageJ}

For analysis of interuser variability of ImageJ, volume measurements of total liver volume, functional liver volume, and tumor volume performed before surgery by two investigators (S.A.W.G.D., J.H.M.B.S.) were compared. The first investigator had 3 years' experience and the second investigator had no experience with the applied software and received instructions and training before starting with the measurements. Bland-Altman plots were used (see below, under Statistical Analysis) to analyze the reliability of the ImageJ method.

\section{Time consumption: ImageJ}

The time consumed to perform one volumetric assessment of a liver with ImageJ including the total liver, the tumor, and the resected specimen was recorded for each of the 15 livers. To assess time consumption, workload, and influence of slice thickness, the time needed to outline these three ROIs (total liver, tumor, resected specimen) was recorded per slice in five livers.

As for any volumetric measurement technique based on CT imaging data sets, the accuracy of the volume measurements is dependent on the slice thickness used for the volume calculation. The slice thickness used for volume measurement is also crucial for the time needed to perform the analysis because of manual outlining. Therefore, the influence of slice thickness on CT-based liver volume measurements using ImageJ was also investigated. Total liver volume (TLV) was analyzed based on different slice thicknesses in these patients undergoing a right hemihepatectomy. The relative change in TLV between volumetry performed with the standard of reference $(0.5 \mathrm{~cm})$ and volumetric measurements using thicker slices $(1.0,1.5$, and $2.0 \mathrm{~cm}$ ) was calculated with the following formulas. $\left(\mathrm{TLV}_{1.0}-\mathrm{TLV}_{0.5}\right) / \mathrm{TLV}_{0.5} \times 100 \% ;\left(\mathrm{TLV}_{1.5}-\mathrm{TLV}_{0.5}\right) /$ $\mathrm{TLV}_{0.5} \times 100 \%$; $\left(\mathrm{TLV}_{2.0}-\mathrm{TLV}_{0.5}\right) / \mathrm{TLV}_{0.5} \times 100 \%$; The relative gain of time between volumetric measurements using different slice thicknesses was recorded and calculated in percentages as well.

Statistical analysis

Pearson's coefficient was used to quantify the association between the volume of the resected specimen and the actual weight of the resection specimen. A value of $P<0.05$ was considered to indicate statistical significance. The resulting regression line was described as a linear 
equation, and the correlation coefficient ( $r$ ) was calculated. Changes of calculated volumes were tested against zero with the use of a Wilcoxon signed rank test with a theoretical mean of zero. Data are presented as the mean \pm standard error of the mean (SEM).

The mean and standard deviation (SD) were calculated from the two volumetric results for each user using ImageJ. Next, the limits of agreement as proposed by Bland and Altman [16] were calculated. This method is based on the mean and SD of the difference between two ratings of the same patient. The mean difference between two users indicates systematic error. The SD of the difference between two users indicates random error. The $95 \%$ limits of agreement as in Bland and Altman's plots result in both quantification of random error and systematic error $(1.96 * \mathrm{SD})$ of the measurement method. Statistics were performed using Prism 5.0 for Windows (GraphPad Software, San Diego, CA, USA).

\section{Results}

\section{Patients}

Fifteen patients scheduled for right hemihepatectomy were included in the study. The median age of the patients was 63 years (48-79 years). None of the patients had preoperative evidence of underlying liver disease, and routine liver function tests were uniformly normal (Table 1).

Relation between prospective CT-measured liver volume and resected liver weight

The mean weight of the resection specimens measured in the operating theater was $788.8 \pm 53.7 \mathrm{~g}$. The mean resected liver volumes calculated with ImageJ, OsiriX ${ }^{\circledR}$, and iNtuition ${ }^{\circledR}$ were $987.7 \pm 64.0,944.7 \pm 64.1$, and $905.0 \pm 59.9 \mathrm{ml}$, respectively. The mean ratios between the measured weight and volume measured by ImageJ, OsiriX $^{\circledR}$, and iNtuition ${ }^{\circledR}$ were $0.78 \pm 0.03,0.81 \pm 0.04$, and $0.83 \pm 0.04$, respectively. The mean remnant liver volume percentages measured by ImageJ, OsiriX ${ }^{\circledR}$, and
iNtuition ${ }^{\circledR}$ were $41.4 \pm 1.6 \%, 36.2 \pm 1.5 \%$, and $37.8 \pm$ $1.6 \%$, respectively (Table 2 ).

In one patient two surgical procedures were possible, either a right posterior sectionectomy or a right hemihepatectomy. Both volumes were calculated prospectively, and finally the volume for right posterior sectionectomy was included in the analysis. There was a significant correlation between the resected liver volumes calculated with ImageJ, OsiriX $^{\circledR}$, and iNtuition ${ }^{\circledR}$ prospectively and the actual measured weights of the resection specimens: $r=0.89$ and $P<0.001 ; r=0.83$ and $P<0.001 ; r=0.85$ and $P<$ 0.001; respectively (Fig. 1).

Interobserver variability: ImageJ

Liver volumetry measurements using ImageJ were compared using Bland-Altman plots. The mean difference of TLV measurements between both users was $49.4 \pm 86.1 \mathrm{ml}$. The $95 \%$ limits of agreement were -218.2 to $119.4 \mathrm{ml}$ (Fig. 2a). The mean difference of the liver resection specimen measurements between both users was $53.0 \mathrm{ml} \pm$ $89.8 \mathrm{ml}$. The $95 \%$ limits of agreement were -228.9 to

Table 1 Patients' characteristics

\begin{tabular}{ll}
\hline Characteristic & Median (range) \\
\hline Age (years) & $63(48-79)$ \\
Sex $(\mathrm{M} / \mathrm{F})$ & $(8 / 7)$ \\
Height $(\mathrm{cm})$ & $170(155-191)$ \\
Weight $(\mathrm{kg})$ & $76(55-127)$ \\
BMI $\left(\mathrm{kg} / \mathrm{m}^{2}\right)$ & $26(19-43)$ \\
AST $(\mathrm{IU} / \mathrm{l})$ & $22(7-52)$ \\
ALT $(\mathrm{IU} / \mathrm{l})$ & $30(8-61)$ \\
LDH $(\mathrm{IU} / \mathrm{l})$ & $372(312-469)$ \\
$\gamma \mathrm{GGT}(\mathrm{IU} / \mathrm{l})$ & $37(29-258)$ \\
ALP $(\mathrm{IU} / \mathrm{l})$ & $98(57-168)$ \\
Bilirubin $(\mu \mathrm{M})$ & $12(7-15)$ \\
\hline
\end{tabular}

$B M I$ body mass index, $A S T$ aspartate aminotransferase, $A L T$ alanine aminotransferase, $L D H$ lactate dehydrogenase, $\gamma G G T$-glutamyltranspeptidase, $A L P$ alkaline phosphatase

Table 2 Results for 15 CT volumetry and intraoperative measurements

\begin{tabular}{|c|c|c|c|c|}
\hline Measurement & ImageJ user 1 & ImageJ user 2 & OsiriX $^{\circledR}$ & iNtuition $^{\circledR}$ \\
\hline Total liver volume $(\mathrm{ml})$ & $1736.0 \pm 104.0$ & $1785.0 \pm 114.9$ & $1536.0 \pm 98.6$ & $1540.0 \pm 99.9$ \\
\hline Resected liver volume (ml) & $987.7 \pm 64.0$ & $1050 \pm 78.6$ & $944.7 \pm 64.1$ & $905.0 \pm 59.9$ \\
\hline Tumor volume (ml) & $14.0 \pm 4.3$ & $18.9 \pm 4.4$ & $12.0 \pm 3.7$ & $12.4 \pm 4.0$ \\
\hline Remnant liver volume (\%) & $41.4 \pm 1.6$ & $39.0 \pm 1.4$ & $36.2 \pm 1.5$ & $37.8 \pm 1.6$ \\
\hline Resected specimen weight/volume ratio $(\mathrm{g} / \mathrm{ml})$ & $0.78 \pm 0.03$ & $0.77 \pm 0.03$ & $0.81 \pm 0.04$ & $0.83 \pm 0.04$ \\
\hline Resected specimen weight $(\mathrm{g})$ & $788.8 \pm 53.7$ & & & \\
\hline
\end{tabular}

$C T$ computed tomography 


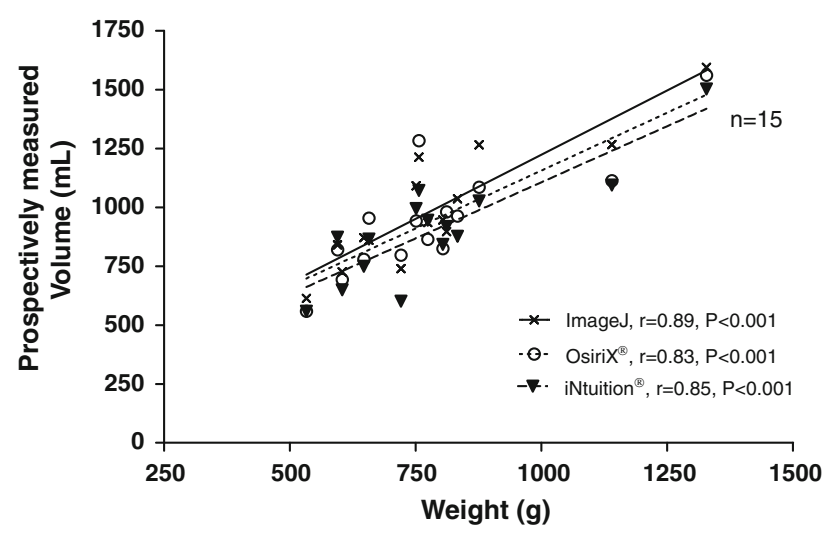

Fig. 1 Correlation between volume measured prospectively with ImageJ, OsiriX ${ }^{\circledR}$, and iNtuition ${ }^{\circledR}$ and the resected specimen's weight measured in the operating theater

$122.9 \mathrm{ml}$ (Fig. 2b). The mean difference of the \%FRLV measurements between both users was $2.3 \pm 4.6 \%$. The $95 \%$ limits of agreement were $-6.7 \%$ to $11.4 \%$ (Fig. 2c).

Time to perform measurement and effect of slice thickness

The time required for a single volumetric assessment of the liver with ImageJ (including total liver, tumor, resected specimen) was, on average, 20 minutes (range 15-30 minutes). There was no statistical difference between the TLVs based on 0.5 vs. $1.0 \mathrm{~cm}, 0.5$ vs. $1.5 \mathrm{~cm}$, and 0.5 vs. $2.0 \mathrm{~cm}$. The change in volume (calculated in percentages) was not significantly different for volumetric measurements with different slice thicknesses. For a slice thickness of 1.0 or $1.5 \mathrm{~cm}, 80 \%$ of the measurements were within the $5 \%$ limit of acceptance for the TLV change. When using the $2.0 \mathrm{~cm}$ slice thickness, only $60 \%$ of the measurements were within this limit. The mean gain of time (calculated in percentages) by increasing slice thickness was $50 \%$ ( 0.5 vs. $1.0 \mathrm{~cm}), 64 \%(0.5$ vs. $1.5 \mathrm{~cm})$, and $72 \%(0.5$ vs. $2.0 \mathrm{~cm})$.

ImageJ versus OsiriX ${ }^{\circledR}$ and iNtuition ${ }^{\circledR}$

There was a highly significant correlation between the volumes calculated with ImageJ and the volumetric analysis using OsiriX ${ }^{\circledR}(r=0.95, P<0.001)$. There was also a strong correlation between the volumes calculated with Image J and the volumetric analysis performed by a radiologist using radiology image analysis software linked to radiology hardware $(r=0.93, P<0.001)$.

\section{Discussion}

The present study shows that prospective CT volumetric analysis of the liver in patients undergoing right
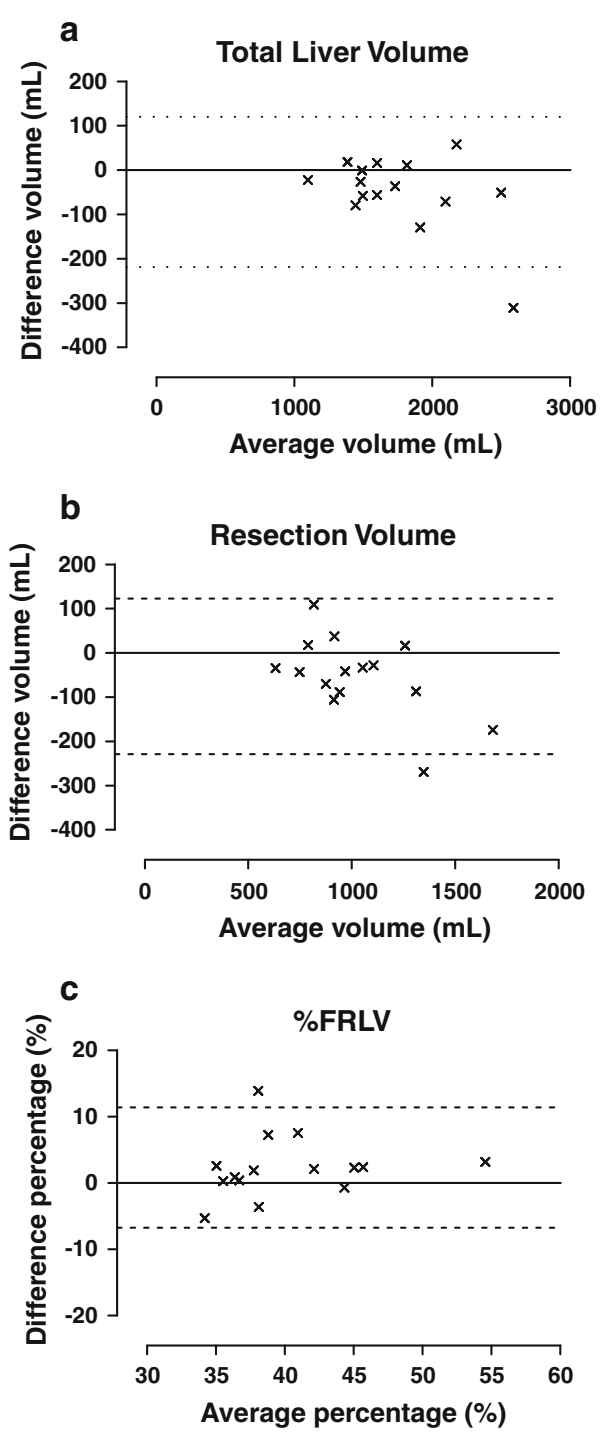

Fig. 2 a Bland-Altman analysis for interobserver variability of outlining the total liver volume with ImageJ. Horizontal solid line indicates the mean average between the two users. Horizontal dashed lines indicate $95 \%$ limits of agreement (mean $\pm 1.96 \mathrm{SD}$ ). b BlandAltman analysis for interobserver variability of outlining the resectional liver volume with ImageJ. c Bland-Altman analysis for interobserver variability of the \%functional remnant liver volume (\%FRLV) with ImageJ

hemihepatectomy with ImageJ and OsiriX ${ }^{\circledR}$ on the surgeon's PC is valid and reliable. In this study, we found a strong significant correlation between resection weight and resection volume measured prospectively with ImageJ and OsiriX ${ }^{\circledR}$. Practical advantages of ImageJ and Osiri $\mathrm{X}^{\circledR}$ were described previously by our group [11, 12]. In our center, the radiologists use the commercial image analysis software package iNtuition ${ }^{\circledR}$ for liver CT volumetry. There was a significant correlation between the resection weight and the resection volume measured prospectively with iNtuition ${ }^{\circledR}$. There were no significant differences in the mean volumes 
between the three software packages. There was a strong correlation between the volumes measured with radiology software iNtuition ${ }^{\circledR}$ and the volumes measured with ImageJ and OsiriX ${ }^{\circledR}$. This indicates that ImageJ and Osiri ${ }^{\circledR}$ even if operated by nonradiologists, are at least as appropriate for CT volumetry of the liver as professional radiology software. ImageJ and Osiri ${ }^{\circledR}$ bring preoperative liver CT volumetry within the surgeon's reach, independent of the support of a radiologist. Hepatopancreatobiliary (HPB) surgeons should routinely perform CT volumetry during the preoperative assessment of patients undergoing a major liver resection. This is especially true for patients with compromised liver function due to neoadjuvant chemotherapy, cirrhosis, or steatosis or for patients scheduled for an extended liver resection.

As we [11] and others [17] concluded before, the time required to perform $\mathrm{CT}$ volumetry of the liver can be reduced by increasing slice thickness, thereby diminishing the number of CT slices that have to be outlined. Data from the current study show that to make a rough estimate of the FRLV (patients with a percentage FRLV of $\geq 30 \%$ ) a slice thicknesses of $1.0 \mathrm{~cm}$ provides an optimal balance between accuracy and time efficiency. However, in patients with a marginally functional liver remnant volume (20-30\%) greater accuracy is needed. In these patients, it is better to use a smaller slice thickness to avoid over- or underestimating the volume. Time required to outline all ROIs is approximately 20 minutes with both ImageJ and OsiriX ${ }^{\circledR}$. However, OsiriX $\mathrm{X}^{\circledR}$ has a significant advantage because it has an integrated picture archiving and communication system (PACS system) in which the data (CT scans) of all examined patients are automatically stored. Additionally, OsiriX ${ }^{\circledR}$ is more user-friendly, and ROIs can be selected semiautomatically, which saves time and lessens the workload [12].

Interobserver variability for ImageJ between the two users for volumetric assessment of the total and resected liver volumes was low. Bland-Altman plots revealed that there was one outperformer in the measurements of the total liver volume and the resection specimen volume. This resulted in relatively wide confidence intervals. However, it is mainly the \%FRLV that is crucial. It has been shown elsewhere that to perform liver resection safely \%FRLV has to be at least $26.6 \%$ in patients with normal liver parenchyma [4]. In the present study, the mean difference of the $\%$ FRLV measurements between both ImageJ users of $2.3 \pm 4.6 \%$ was clinically acceptable because ImageJ users 1 and 2 predicted \%FRLVs of $41.4 \pm 1.6 \%$ and $39.0 \pm$ $1.4 \%$, respectively. In other words, the resection volume differences between the two ImageJ users should have had no clinical consequences. In patients in whom the preoperative liver volume assessment shows that the reported FRLV\% approaches $26.6 \%$, extra care is recommended.
A possible limitation of estimating the future remnant liver volume with $\mathrm{CT}$ volumetry is the systematic overestimation of the liver volumes compared to the resected specimens' weight. A physical density $<1.0 \mathrm{~g} / \mathrm{ml}$ and systematic overestimation of the liver volume in the present study was in accordance with those of previous studies [7, 10-12]. Inaccurate estimation of the resected specimen volume by $\mathrm{CT}$ volumetry can occur because the liver on a CT scan is in a perfused state and the postoperative resection specimen is in an unperfused state. This applies to every radiologic application, and therefore some studies have suggested the implementation of conversion factors to improve volumetric accuracy $[7,18,19]$. These factors are mostly based on the intraoperative resection volume determined with the use of Archimedes principle. Unfortunately, this approach does not determine the influence of the blood content of the resection specimen. It might be that these conversion factors can lead to a more precise volumetric analysis of the liver, but their exact role remains to be determined. Patients with steatosis have more fat in the liver, and the relative densitie of fat is $0.9 \mathrm{~g} / \mathrm{ml}$. Hence, the volume is larger and the weight less than that of a normal liver. Ultimately, this changes the volume/weight standard and can also contribute to systemic overestimation.

In all right hemihepatectomies in the present study, virtual transection followed Cantlie's line, and thus the middle hepatic vein (MHV) was used as a guide for prospective volume measurements. During surgery, the demarcation line after selective inflow occlusion is used for transection and may leave liver parenchyma outside (i.e., to the right of) the MHV in some patients. As a consequence, preoperative estimation of the future resected specimen volume may be overestimated in some individuals. In line with this, Karlo et al. showed in a retrospective study that, despite a highly accurate definition of the transection line on preoperative CT or MRI scans using anatomic landmarks on postoperative MRI scans, the resection specimen volume was still overestimated [7]. Systematic overestimation, however, does not affect the functional remnant liver volume as a percentage of the total functional liver volume, which is one of the most important indicators of the risk of postoperative liver failure.

\section{Conclusions}

User-friendly, easily accessible, free, downloadable software packages such as ImageJ and OsiriX ${ }^{\circledR}$ can be used reliably for prospective CT volumetry of resected liver specimens. The performance of ImageJ and OsiriX ${ }^{\circledR}$ is comparable to that of radiology software iNtuition ${ }^{\circledR}$. OsiriX $^{\circledR}$ is the most efficient and practical software 
package, but it can be used only on a Macintosh PC. For a Windows-based PC, ImageJ is a good alternative.

Acknowledgments The authors thank Marc H. A. Bemelmans, Steven W. M. Olde Damink, and Marcel C. G. van de Poll for their contributions to the conception and design of the study and for technical assistance. Simon A. W. G. Dello was supported by a Kootstra Talent Fellowship from Maastricht University.

Open Access This article is distributed under the terms of the Creative Commons Attribution Noncommercial License which permits any noncommercial use, distribution, and reproduction in any medium, provided the original author(s) and source are credited.

\section{References}

1. Shah SA, Haddad R, Al-Sukhni W et al (2006) Surgical resection of hepatic and pulmonary metastases from colorectal carcinoma. J Am Coll Surg 202:468-475

2. Fusai G, Davidson BR (2003) Management of colorectal liver metastases. Colorectal Dis 5:2-23

3. Scheele J, Altendorf-Hofmann A, Grube T et al (2001) Resection of colorectal liver metastases: what prognostic factors determine patient selection? Chirurg 72:547-560

4. Schindl MJ, Redhead DN, Fearon KC et al (2005) The value of residual liver volume as a predictor of hepatic dysfunction and infection after major liver resection. Gut 54:289-296

5. Shoup M, Gonen M, D'Angelica M et al (2003) Volumetric analysis predicts hepatic dysfunction in patients undergoing major liver resection. J Gastrointest Surg 7:325-330

6. Jarnagin WR, Gonen M, Fong Y et al (2002) Improvement in perioperative outcome after hepatic resection: analysis of 1,803 consecutive cases over the past decade. Ann Surg 236:397-407

7. Karlo C, Reiner CS, Stolzmann P et al (2010) CT- and MRIbased volumetry of resected liver specimen: comparison to intraoperative volume and weight measurements and calculation of conversion factors. Eur J Radiol 75:107-111
8. Rasband W (1997-2005) ImageJ, 1.33 edn. National Institutes of Health, Bethesda

9. Kishi Y, Abdalla EK, Chun YS et al (2009) Three hundred and one consecutive extended right hepatectomies: evaluation of outcome based on systematic liver volumetry. Ann Surg, Aug 27 [Epub ahead of print]

10. Wigmore SJ, Redhead DN, Yan XJ et al (2001) Virtual hepatic resection using three-dimensional reconstruction of helical computed tomography angioportograms. Ann Surg 233:221-226

11. Dello SA, van Dam RM, Slangen JJ et al (2007) Liver volumetry plug and play: do it yourself with ImageJ. World J Surg 31:2215-2221

12. Van der Vorst JR, van Dam RM, van Stiphout RS et al (2010) Virtual liver resection and volumetric analysis of the future liver remnant using open source image processing software. World J Surg 34:2426-2433

13. Lu Y, Wu Z, Liu C, Wang HH (2004) Hepatic volumetry with PhotoShop in personal computer. Hepatobiliary Pancreat Dis Int 3:82-85

14. Tu R, Xia LP, Yu AL et al (2007) Assessment of hepatic functional reserve by cirrhosis grading and liver volume measurement using CT. World J Gastroenterol 13:3956-3961

15. Dejong CHC, Garden OJ (2003) Neoplasms of the liver. In: Majid AA, Kingsnorth A (eds) Advanced surgical practice. Greenwich Medical Media, London, pp 146-156

16. Bland JM, Altman DG (1995) Comparing methods of measurement: why plotting difference against standard method is misleading. Lancet 346:1085-1087

17. Reiner CS, Karlo C, Petrowsky H et al (2009) Preoperative liver volumetry: how does the slice thickness influence the multidetector computed tomography- and magnetic resonance-liver volume measurements? J Comput Assist Tomogr 33:390-397

18. Lemke AJ, Brinkmann MJ, Schott T et al (2006) Living donor right liver lobes: preoperative CT volumetric measurement for calculation of intraoperative weight and volume. Radiology 240:736-742

19. Hwang S, Lee SG, Kim KH et al (2002) Correlation of blood-free graft weight and volumetric graft volume by an analysis of blood content in living donor liver grafts. Transplant Proc 34: 3293-3294 